\title{
Visual symptomatology and referral patterns for Operation Iraqi Freedom and Operation Enduring Freedom veterans with traumatic brain injury
}

\author{
Ryan Bulson, OD, MS, FAAO $;^{1-2 *}$ Weon Jun, OD, FAAO; ${ }^{1-2}$ John Hayes, PhD ${ }^{2}$ \\ ${ }^{1}$ Portland Department of Veterans Affairs Medical Center, Portland, OR; ${ }^{2}$ Pacific University College of Optometry, \\ Forest Grove, OR
}

\begin{abstract}
Advances in protective armor technology and changes in the "patterns of war" have created a population of Operation Iraqi Freedom/Operation Enduring Freedom (OIF/ $\mathrm{OEF}$ ) veterans with traumatic brain injury (TBI) that provide a unique challenge to Department of Veterans Affairs (VA) healthcare practitioners. The purpose of the study was to determine the frequency of symptomatic ocular and visual sequelae of TBI in OIF/OEF veterans at the Portland VA Medical Center, a Polytrauma Support Clinic Team site. A retrospective analysis of $100 \mathrm{OIF} / \mathrm{OEF}$ veterans with TBI was conducted to determine the prevalence of ocular and visual complaints. Referral patterns were also investigated. Visual symptoms were reported in approximately $50 \%$ of veterans with TBI. Loss of consciousness, but not number of deployments or number of blast exposures, was found to have a statistically significant association with severity of reported visual symptoms. The most commonly reported symptoms included blurred vision (67\%), photosensitivity (50\%), and accommodative problems (40\%). Visual symptoms of OIF/OEF veterans at the Portland VA Medical Center are reported at slightly lower rates than similar studies conducted at the Palo Alto and Edward Hines Jr VA facilities.
\end{abstract}

Key words: Neurobehavioral Symptom Inventory, Operation Enduring Freedom, Operation Iraqi Freedom, postconcussive syndrome, postdeployment, posttraumatic stress disorder, symptom, traumatic brain injury, veteran, vision.

\section{INTRODUCTION}

The high incidence of nonpenetrating injuries from explosive blasts has made mild traumatic brain injury (TBI) the "signature wound" of Operation Iraqi Freedom (OIF) and Operation Enduring Freedom (OEF) [1]. Estimates state that 10 to 20 percent of $\mathrm{OIF} / \mathrm{OEF}$ veterans return from the theater of operations having sustained a TBI [2]. Improvised explosive devices (IEDs) have been estimated to be responsible for approximately 60 percent of these TBIs, as well as 40 percent of Coalition casualties [3-4]. TBIs from IEDs can occur through several mechanisms. Primary blast injuries include those sustained from the initial blast wave itself. The extreme pressure changes associated with the blast wave tend to preferentially damage organs with air-fluid interfaces,

\footnotetext{
Abbreviations: IED $=$ improvised explosive device, NSI $=$ Neurobehavioral Symptom Inventory, OEF = Operation Enduring Freedom, OIF $=$ Operation Iraqi Freedom, PNS $=$ Polytrauma Network Site, PRC = Polytrauma Rehabilitation Center, PSCT = Polytrauma Support Clinic Team, PTSD = posttraumatic stress disorder, TBI = traumatic brain injury, VA = Department of Veterans Affairs, VHA = Veterans Health Administration.

*Address all correspondence to Ryan Bulson, OD, MS, FAAO; 2043 College Way, Jefferson Hall, Forest Grove, OR 97116; 503-352-2830; fax: 503-352-2261.

Email: ryan.bulson@pacificu.edu

http://dx.doi.org/10.1682/JRRD.2011.02.0017
} 
such as rupturing of the tympanic membranes. A secondary injury occurs as a result of propulsion of shrapnel within the radius of the blast exposure. A tertiary injury occurs when an individual is physically displaced from his or her position either to the ground or into a stationary object. Finally, the heat wave generated by the blast may result in burns or exposure to noxious chemicals: a quaternary injury [4].

In April 2007, the Department of Veterans Affairs (VA) issued a directive requiring all $\mathrm{OIF} / \mathrm{OEF}$ veterans to be screened for TBI. This screening includes a military history questionnaire and the Neurobehavioral Symptom Inventory (NSI). The goal of these surveys is to ascertain a history of potentially traumatic events sustained in the combat zone, including exposure to blasts, explosions, and artillery, as well as symptoms immediately after such traumatic events and persistence of these symptoms into the present. A positive screening results in a referral to the appropriate provider for additional clinical evaluation $[1,5]$.

The Polytrauma System of Care was designed to manage veterans diagnosed with TBI [6]. A summary of the Polytrauma System of Care is available from http:// www.polytrauma.va.gov/system-of-care/care-facilities/. Polytrauma Rehabilitation Centers (PRCs) are inpatient facilities for the most severe injuries and are located in Minneapolis, Minnesota; Palo Alto, California; Richmond, Virginia; and Tampa, Florida. Regional Polytrauma Network Sites (PNSs) provide the second tier of care by managing postacute injuries; there are 21 such sites across the country. Finally, 130 Polytrauma Support Clinic Teams (PSCTs) consist of interdisciplinary teams that manage postacute sequelae in an outpatient setting and make referrals to the PRCs and PNSs as appropriate. The Portland VA Medical Center serves as one of these PSCTs.

In addition to these general screenings, Veterans Health Administration (VHA) Directive 065, issued October 2008, states "every prior (since February 2005), current, and future patient with a diagnosis of TBI admitted to a VA PRC must have a TBI specific ocular health and visual functioning eye examination performed by an optometrist or ophthalmologist" [7] (available online from http://wwwl.va.gov/ vhapublications/ViewPublication.asp?pub ID $=1783$ ).

The tests required under this directive are extensive and apply specifically to PRCs. This directive has been successful in evaluating and managing veterans with the most severe TBIs, as well as spreading public awareness about the ocular and visual consequences of TBI.

The ocular and visual sequelae of TBI in the general population have been well documented. Anomalies of the accommodative, binocular, and oculomotor systems, as well as vestibular dysfunction and perceptual and cognitive delays, are often reported following TBI [8]. Visual field defects, photosensitivity, and ocular surface disease are also common ocular health sequelae of mild TBI [9]. In populations recovering from TBI, the prevalence of these visual disturbances has been reported as ranging from 30 to 85 percent [10]. Recent studies have described the occurrence of visual and vestibular symptoms in OIF/ OEF veterans with TBI. Stelmack et al. reported that visual symptoms were self-reported in 75 percent of OIF/ OEF veterans with TBI at the Edward Hines Jr VA Hospital (PNS) [11]. In a retrospective study of the Palo Alto VA (PRC) healthcare system, Brahm et al. found that 75.4 percent of OIF/OEF veterans at PRCs reported subjective visual complaints [12]. For OIF/OEF veterans with presumed TBI being seen at the center's outpatient PNS, 75.8 percent of patients reported a subjective visual complaint. An earlier study of polytrauma patients at the Palo Alto VA (PRC) revealed self-reported visual symptoms in 74 percent of the population [13]. Similarly, Lew et al. observed that 75 percent of veterans with TBI reported visual symptoms at the Palo Alto VA (PNS) [5]. Thus, it is clear that OIF/OEF veterans with TBI are very likely to complain of visual symptoms during screenings.

The goal of the present study is to determine the frequency of self-reported ocular and visual symptoms in OIF/OEF veterans with TBI at the Portland VA Medical Center, a PSCT. Further, this study will examine the referral patterns to the eye care team to determine whether visual symptoms are being adequately addressed in this population.

\section{METHODS}

We reviewed 185 records from OIF/OEF veterans examined in the postdeployment clinic from January 2009 to the present. Patient records were included in the present study if a diagnosis of TBI was given at the initial postdeployment evaluation with the medical doctor. The records of those OIF/OEF veterans who were evaluated in the postdeployment clinic by a medical doctor but not given a diagnosis of TBI were excluded. The present 
study examines $100 \mathrm{OIF} / \mathrm{OEF}$ veteran records with a diagnosis of TBI based on the postdeployment evaluation. The information from each electronic medical record was extracted, de-identified, and entered into a secure database. Specific information from each veteran's initial evaluation by a medical doctor in the postdeployment clinic was reviewed. These records included a military history questionnaire eliciting specific information about blast exposures capable of inducing a TBI during deployment, as well as the NSI to determine the severity of neurological and psychological symptoms that have caused disturbance since deployment. For the purposes of this study, a score of 2 or greater out of 4 on the NSI was considered to be significant. This level corresponded to symptoms of moderate or greater intensity and was determined by Stelmack et al. to have an adverse effect on daily activities [11]. Statistical analysis was performed using SPSS for descriptive statistics, correlations, and $t$-tests (PASW v18, IBM; Armonk, New York) and Linacre (v3.66, Winsteps; Berkeley, California) for Rasch analysis.

\section{RESULTS}

The mean age of veterans in this study was $29.9 \mathrm{yr}$ (range: 21-55 yr), and 99 percent of veterans were male. Of the records studied, 59 percent of these veterans reported a single deployment, while two, three, and four or greater deployments were reported by 28 percent, 10 percent, and 3 percent of subjects, respectively. Multiple blast injuries accounted for the majority (69\%) of TBIs, with the other causes being blasts associated with motor vehicle accidents (13\%), single blasts (10\%), falls $(7 \%)$, and isolated motor vehicle accidents (1\%). Loss of consciousness was reported by 27 percent of subjects.

The frequencies of reported symptoms on the NSI in the present study are compared with those of Stelmack et al. in Figure 1 [11]. A score of 2 or greater was reported by 47 percent of veterans for "Blur/Trouble Seeing" compared with 63 percent in the Stelmack et al. study, while 54 percent responded with a score of 2 or greater for "Light Sensitivity" compared with 59 percent per Stelmack et al. Figure 1 also compares the frequencies compiled in the

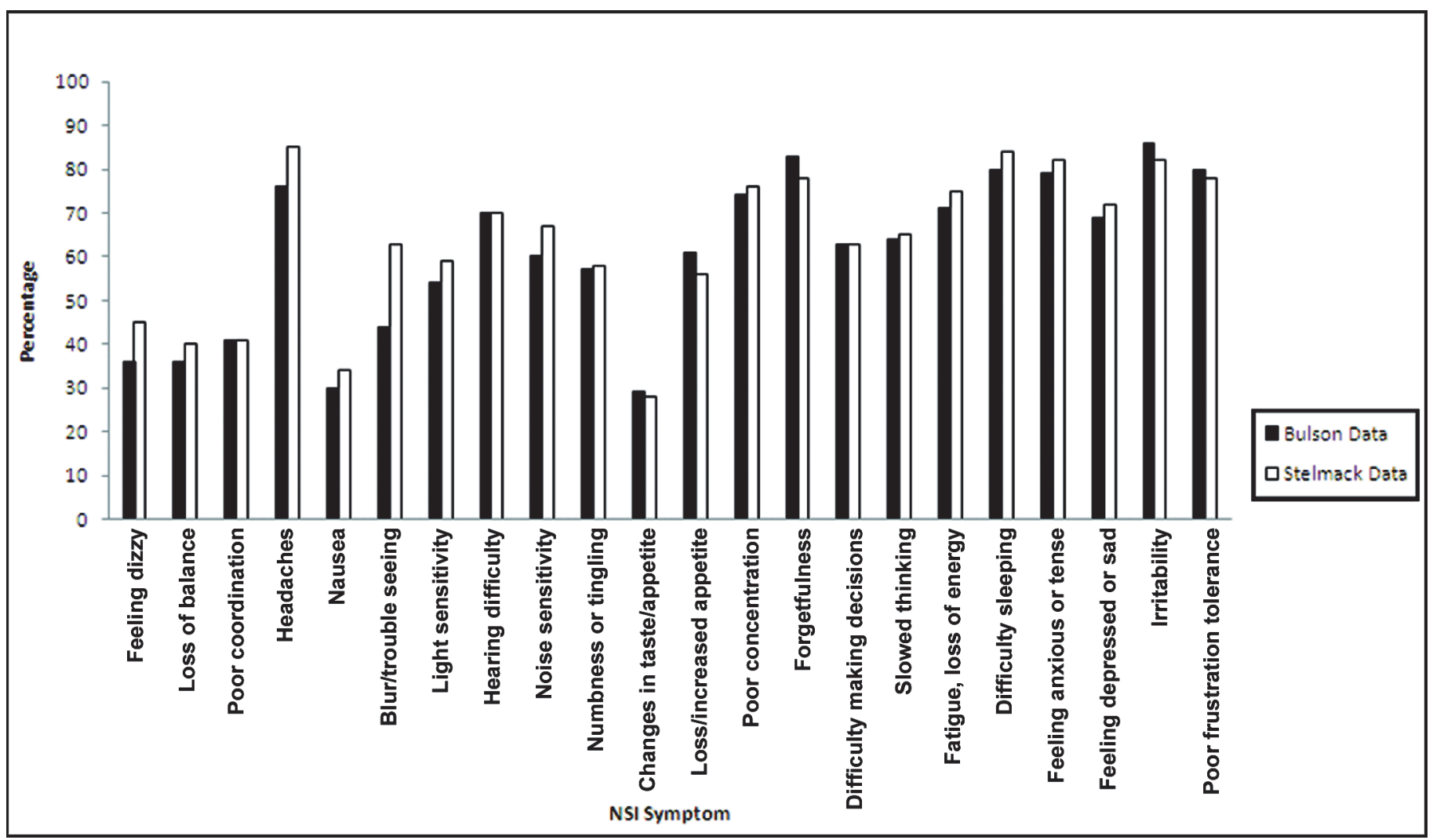

Figure 1.

Percentage reporting Neurobehavioral Symptom Inventory (NSI) score of $\geq 2$ : comparison of present study versus Stelmack et al. [11]. 
present study with those reported by Stelmack et al. [11]. Only the frequency of reported problems with "Blur/Trouble Seeing" were statistically different from the findings of Stelmack et al. via Chi square analysis $\left(\chi^{2}=5.71, p=\right.$ 0.02).

Rasch analysis was used to provide composite scores for the NSI. The overall single factor scale had Item separation $=3.5$ and Person separation $=5.94$, with reliabilities equal to 0.92 and 0.97 , respectively. Four itemsdifficulty sleeping, numbness or tingling, feeling anxious or tense, and poor coordination - had infit scores $>2$. The outlier questions were not deleted from the scale since a subscale created with them correlated $(r=0.75)$ with the reduced total scale score. The composite Rasch measure was used to test the associations with other variables.

There were no significant correlations between severity of symptoms reported on the NSI and number of blasts $(t=1.56, p=0.12)$ or number of deployments $(r=$ $-0.04, p=0.67)$. Loss of consciousness was determined to be statistically correlated with more severe symptoms on the NSI $(t=3.92, p<0.001)$. A comparison of NSI symptom scores between veterans reporting loss of con- sciousness and those reporting no loss of consciousness is shown in Figure 2.

During the postdeployment evaluation with the primary care physician, 33 percent of veterans self-reported a visual problem. The most commonly self-reported visual symptoms during the initial postdeployment evaluation were blurred vision (67\%), photosensitivity (50\%), focusing problems (40\%), peripheral vision defects $(17 \%)$, tracking problems $(10 \%)$, and double vision $(7 \%)$. Of those veterans reporting visual symptoms on either the NSI or during the postdeployment evaluation, 91 percent were referred to the eye clinic; however, 23 percent of these veterans "no-showed" for their evaluation in the eye clinic.

Of the visually symptomatic veterans examined in the eye clinic, 96 percent had best corrected visual acuities of $20 / 20$ or better. The most common diagnoses of those veterans evaluated in the eye clinic were uncorrected refractive error (96\%), photosensitivity (22\%), accommodative dysfunction $(13 \%)$, dry eye syndrome $(9 \%)$, and visual field defect (9\%).

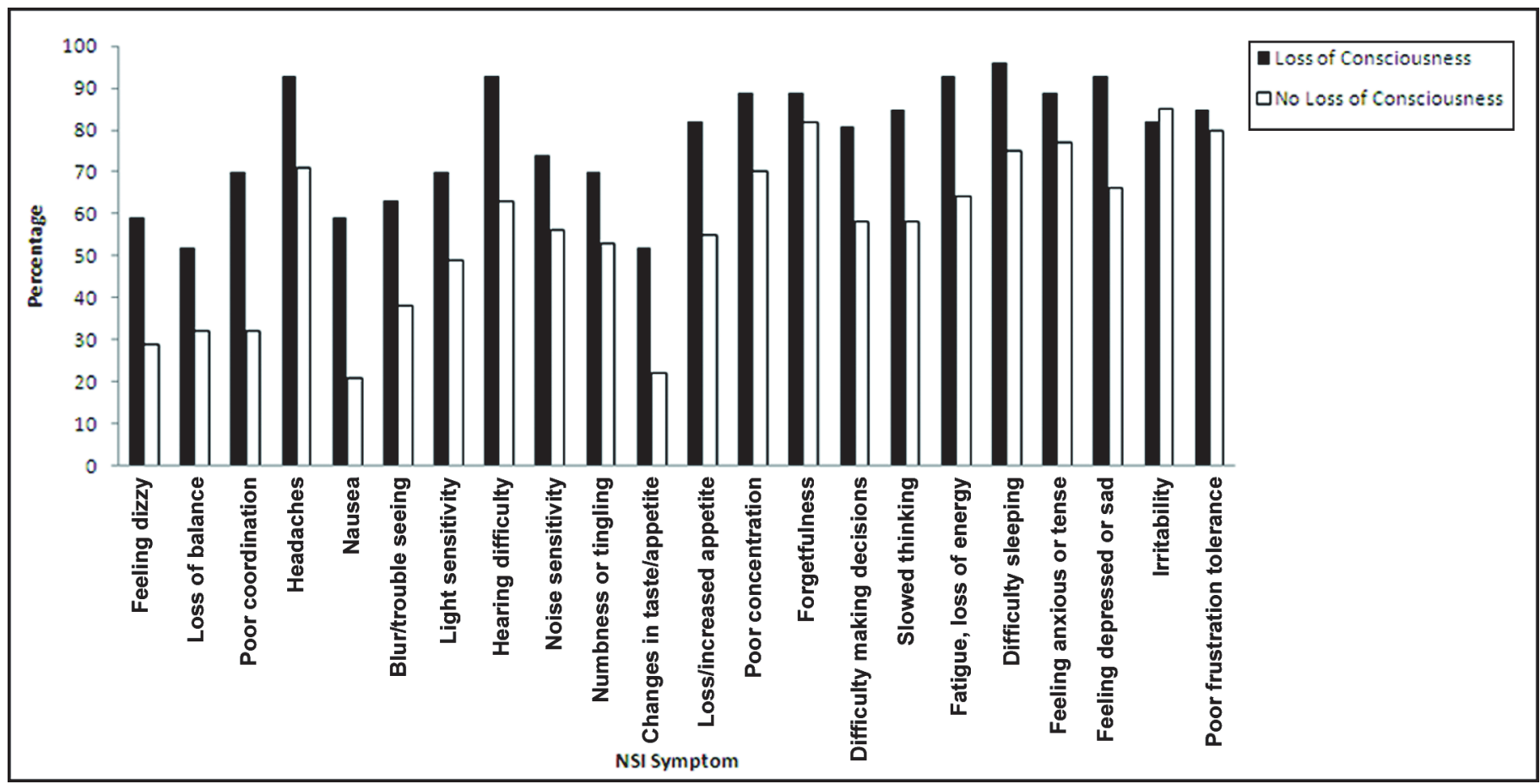

Figure 2.

Percentage reporting Neurobehavioral Symptom Inventory (NSI) score of $\geq 2$ : comparison of reported loss of consciousness. 


\section{DISCUSSION}

Self-reported visual symptoms in OIF/OEF veterans with TBI at the Portland VA Medical Center (PSCT) occurred with a frequency of 44 to 54 percent via the NSI and 33 percent during the initial postdeployment evaluation. Previous studies at the Palo Alto VA Health Care System (PRC) and Edward Hines Jr VA Hospital (PNS) have consistently reported visual symptoms with a frequency of approximately 75 percent $[5,11-13]$. The discrepancy observed in the current study is likely related to the VA polytrauma rehabilitation hierarchy discussed in the "Introduction" section. The Palo Alto VA Health Care System includes both a PRC and PNS, while the Edward Hines Jr VA Hospital features a PNS. The Portland VA Health Care System serves as a PSCT managing the least severe TBI patients in an outpatient setting, which is one explanation for the lower incidence of self-reported symptoms in the Portland VA population. Interestingly, in comparing the NSI symptom frequencies with those reported by Stelmack et al. [11], the only significant difference observed was a higher frequency of "Blur/Trouble Seeing" at the Edward Hines Jr VA PNS. It is not entirely clear why veterans at a PNS, a higher tier on the Polytrauma System of Care than the Portland VA Medical Center, would report a higher frequency of this visual symptom but no other symptoms on the NSI. Based on the Polytrauma System of Care, veterans at a PNS are generally recovering from more severe injuries than those at a PSCT, so it is possible that the ocular and visual sequelae in this population are having a greater effect on quality of life than other symptoms and are therefore reported at higher rates on the NSI.

Among the most frequently reported visual symptoms at the Portland VA Medical Center were blurred vision and focusing problems. The most common etiologies for these visual complaints were uncorrected refractive error and accommodative dysfunction. Of those veterans that were referred to the eye care team, 96 percent were found to have some type of uncorrected refractive error. Given the young demographic of OIF/OEF veterans in this study (mean age $29.9 \mathrm{yr}$ ), accommodative dysfunction likely contributed to symptoms of blurred vision and problems focusing. Accommodation refers to the ability of the eye to form and maintain a focused retinal image on an object of interest. The frequency of occurrence of accommodative dysfunction in prepresbyopic patients with TBI ranges from approximately 10 to 40 percent [8]. The most common dysfunctions of accommodation include accommodative insuf- ficiency (underaccommodation), accommodative excess/ spasm (overaccommodation), and accommodative infacility (inflexibility of accommodation) [10]. Green et al. recently investigated both the static and dynamic accommodation levels in a population of patients with mild TBI [14]. Accommodative responses, including both monocular and binocular accommodative amplitude, accommodative convergence to accommodation ratio, and negative and positive relative accommodation, were reduced in the TBI population compared with normative values. In addition, the TBI cohort also exhibited a significant fatigue effect during accommodative facility testing, which is atypical in the non-TBI population. Thus, reported problems with blurred vision and focusing problems may have been related to both uncorrected refractive error and accommodative dysfunction.

A statistically significant association was observed between severity of symptoms on the NSI and reported loss of consciousness. These findings are not surprising given that loss of consciousness is often used to classify the severity of a TBI $[3,15]$. Hoge et al. observed that veterans with TBI reporting loss of consciousness were at a significantly higher risk for developing physical and mental health problems than those who did not lose consciousness [2]. Furthermore, 40 percent of these veterans met the criteria for posttraumatic stress disorder (PTSD). Thus, it is clear that veterans with TBI associated with loss of consciousness are likely to develop more severe posttraumatic symptoms. Note, however, that symptoms were also reported at relatively high rates even in those veterans not reporting loss of consciousness.

The findings of the present study indicate that no significant correlation exists between severity of symptoms on the NSI and number of deployments. Previous studies have demonstrated a statistically significant correlation between prevalence of PTSD and both duration and number of deployments [16-17]. In contrast, a more recent study by Fear et al. revealed no association between number of deployments and PTSD, mental health disorders, or alcohol misuse [18]. In agreement with the latter study, one may expect to find a lack of association between severity of symptoms and number of deployments given that the NSI questionnaire used in the present study includes many symptoms of PTSD. Indeed, recent reports have acknowledged that the overlap of symptoms between PTSD and TBI, as well as the commonality of their comorbidity, can make differentiating the two conditions clinically challenging $[4,15]$. 
No significant association was observed between severity of symptoms on the NSI and number of blasts. One possible explanation for this finding is that veterans may be overestimating the number of blasts to which they have been exposed. A similar possibility is that veterans reporting exposure to multiple blasts were within sight or sound of multiple blasts but outside the radius capable of inducing a TBI. It is not clear whether exposure to several of these less forceful, nontraumatic blasts is capable of producing significant long-term injury [4]. If the veterans were indeed exposed to multiple traumatic blasts, the resulting injuries may have caused a disconnect from true symptoms, thereby masking the severity of NSI symptoms. A recent report demonstrated that specific regions of the brain may be more susceptible to blast injuries, specifically areas of the prefrontal cortex near the orbital sockets and nasal sinuses responsible for integration of information from subcortical structures, as well as disinhibition of responses to emotions such as fear and anxiety [19]. Damage to these regions may cause inappropriate activation of normal physiological systems, resulting in cognitive misperception of a person's environment and their symptoms in day to day life.

Nearly 10 percent of visually symptomatic veterans were not referred to the eye clinic for further evaluation. Perhaps more concerning is that 23 percent of visually symptomatic veterans no-showed for their consultations with the eye clinic. The average no-show rate for the Portland VA eye clinic is below 10 percent. A number of causes are likely to blame for a higher no-show rate. First, OIF/OEF veterans are generally a younger demographic than the typical VA population. As a result, these veterans likely have more time demands, including work and family obligations, that make scheduling difficult. Indeed, previous surveys in this population have shown lack of availability because of work schedules [2]. Second, in the present study 83 percent of veterans reported a score of 2 or greater on the NSI category for "forgetfulness." Cognitive deficits and memory problems are welldocumented long-term sequelae of TBI [15]. Konrad et al. recently investigated a comprehensive battery of neuropsychological testing in subjects with TBI [20]. Compared with controls, the TBI group performed statistically worse in assessments of learning, recall, working memory, attention, and executive function. Another recent study demonstrated a significant inverse relationship between severity of TBI and declarative memory performance [21]. The authors used functional magnetic reso- nance imaging studies to determine that reduced medial temporal lobe functionality may be contributing to the memory deficits. Given these findings, it is reasonable to posit that traditional recall systems may not be adequate for this population because of the high frequency of cognitive deficits and memory problems. A final explanation for the high no-show rate of veterans with TBI is the necessity of multiple specialty providers to manage the complex nature of their injuries. These veterans may require multiple appointments each week, potentially resulting in considerable time and financial expense as they are simultaneously working to assimilate back into civilian culture. This is likely to be more problematic for this younger demographic for the reasons previously stated.

The most commonly reported ocular and visual conditions in the current study were uncorrected refractive error (96\%), photosensitivity (22\%), and accommodative dysfunction (13\%). While these conditions were reported at relatively high rates, ocular and visual conditions in this population may have been underrepresented because of the lack of a standardized protocol for evaluating patients with TBI by the eye care team at the Portland VA Medical Center. The Portland VA eye care team TBI template was modeled from VHA Directive 2008-065, which outlines a standardized protocol for evaluating patients with TBI at PRCs; however, this template was not universally accepted and implemented by the eye care team at the time of the study [7]. Optometric evaluations at the Portland VA Medical Center were problem-oriented such that they addressed the patient's primary complaint. The baseline optometric examination included corrected distance visual acuity, corrected near visual acuity, distance and near cover test, extraocular motilities, pupil testing, confrontation visual fields, distance refraction, anterior segment evaluation, intraocular pressure measurement, and dilated fundus examination. Patients presenting to the eye clinic after referral from the postdeployment clinic with a binocular vision-related complaint, for example intermittent blurred vision, blurred vision at near, diplopia, and/or slow focusing, had additional binocular vision testing performed. This testing was conducted at the discretion of the attending optometrist and may have included additional accommodative testing (positive/negative relative accommodation, fused cross cylinder, accommodative amplitudes, accommodative facility) and vergence testing (distance and near lateral and vertical phorias, distance and near convergence and divergence ranges, vertical vergence ranges). 
Because the individual binocular testing varied from provider to provider, it is difficult to determine whether the evaluations reviewed in the present study were adequately identifying binocular vision issues. Thus, a future goal at the Portland VA Medical Center is for all OIF/OEF veterans with confirmed TBI to be evaluated per the testing recommended by VHA Directive 2008-065. In addition, the present study demonstrates a need for standardized testing batteries such as that described by VHA Directive 2008065 for this population at all PNS and PSCT sites.

\section{CONCLUSIONS}

OIF/OEF veterans at the Portland VA Medical Center, a PSCT site, generally reported visual symptoms at lower rates than those veterans at PRCs and PNSs in Palo Alto and Edward Hines Jr VA medical centers. Loss of consciousness was found to be the best predictor for severity of symptoms reported on the NSI, while number of deployments and number of blast exposures were not correlated with severity of symptoms. Most veterans reporting visual symptoms on either the NSI or during their initial postdeployment evaluation were appropriately referred to the eye clinic and, of those who presented to the eye clinic, the vast majority were deemed to have had their visual concerns addressed by the eye care team. However, OIF/OEF veterans with TBI no-showed at double the rate of the normal VA eye clinic population, indicating that traditional VA protocols, including screening batteries, locations, hours, and recall methods, may not adequately address the needs of this population.

\section{ACKNOWLEDGMENTS}

\author{
Author Contributions: \\ Study concept and design: R. Bulson, W. Jun. \\ Acquisition of data: R. Bulson, W. Jun. \\ Analysis and interpretation of data: R. Bulson, W. Jun, J. Hayes. \\ Drafting of manuscript: R. Bulson, W. Jun. \\ Critical revision of manuscript for important intellectual content: \\ R. Bulson, W. Jun. \\ Statistical analysis: J. Hayes. \\ Administrative, technical, or material support: R. Bulson, W. Jun. \\ Study supervision: R. Bulson, W. Jun.
}

Financial Disclosures: The authors have declared that no competing interests exit.

Funding/Support: The study was unfunded at time of manuscript preparation.
Institutional Review: The present study was approved by the Portland VA Medical Center Institutional Review Board. Written informed consent was waived because the study was a retrospective chart review.

\section{REFERENCES}

1. Lew HL. Rehabilitation needs of an increasing population of patients: Traumatic brain injury, polytrauma, and blastrelated injuries. J Rehabil Res Dev. 2005;42(4):xiii-xvi. [PMID:16320135] http://dx.doi.org/10.1682/JRRD.2005.01.0124

2. Hoge CW, McGurk D, Thomas JL, Cox AL, Engel CC, Castro CA. Mild traumatic brain injury in U.S. soldiers returning from Iraq. N Engl J Med. 2008;358(5):453-63. [PMID: 18234750] http://dx.doi.org/10.1056/NEJMoa072972

3. Lew HL, Garvert DW, Pogoda TK, Hsu PT, Devine JM, White DK, Myers PJ, Goodrich GL. Auditory and visual impairments in patients with blast-related traumatic brain injury: Effect of dual sensory impairment on Functional Independence Measure. J Rehabil Res Dev. 2009;46(6): 819-26. [PMID:20104405]

http://dx.doi.org/10.1682/JRRD.2008.09.0129

4. Elder GA, Cristian A. Blast-related mild traumatic brain injury: mechanisms of injury and impact on clinical care. Mt Sinai J Med. 2009;76(2):111-18. [PMID:19306373] http://dx.doi.org/10.1002/msj.20098

5. Lew HL, Poole JH, Vanderploeg RD, Goodrich GL, Dekelboum S, Guillory SB, Sigford B, Cifu DX. Program development and defining characteristics of returning military in a VA Polytrauma Network Site. J Rehabil Res Dev. 2007; 44(7):1027-34. [PMID:18075959] http://dx.doi.org/10.1682/JRRD.2007.05.0073

6. Care facilities-VA polytrauma system of care [Internet]. Washington (DC): Department of Veterans Affairs; 2012 [updated 2012 May 24; cited 2011 Apr 19]. Available from: http://www.polytrauma.va.gov/system-of-care/care-facilities/

7. Veterans Health Administration. Performance of traumatic brain injury specific ocular health and visual functioning examinations for polytrauma rehabilitation centers. Washington (DC): Department of Veterans Affairs; 2008. VHA Directive 2008-065.

8. Kapoor N, Ciuffreda K. Vision deficits following acquired brain injury. In: Cristian A, editor. Medical management of adults with neurologic disabilities. New York (NY): Demos Medical Publishing; 2009. p. 407-23.

9. Han $\mathrm{MH}$. the role of the neuro-rehabilitation optometrist. In: Elbaum J, Benson D, editors. Acquired brain injury: an integrative neuro-rehabilitation approach. Manhassat (NY): Springer; 2007. p. 146-75. 
10. Kapoor N, Ciuffreda KJ. Vision disturbances following traumatic brain injury. Curr Treat Options Neurol. 2002; 4(4):271-80. [PMID:12036500] http://dx.doi.org/10.1007/s11940-002-0027-z

11. Stelmack JA, Frith T, Van Koevering D, Rinne S, Stelmack TR. Visual function in patients followed at a Veterans Affairs polytrauma network site: an electronic medical record review. Optometry. 2009;80(8):419-24.

[PMID:19635432]

http://dx.doi.org/10.1016/j.optm.2009.02.011

12. Brahm KD, Wilgenburg HM, Kirby J, Ingalla S, Chang CY, Goodrich GL. Visual impairment and dysfunction in combat-injured servicemembers with traumatic brain injury. Optom Vis Sci. 2009;86(7):817-25. [PMID:19521270] http://dx.doi.org/10.1097/OPX.0b013e3181adff2d

13. Goodrich GL, Kirby J, Cockerham G, Ingalla SP, Lew HL. Visual function in patients of a polytrauma rehabilitation center: A descriptive study. J Rehabil Res Dev. 2007;44(7): 929-36. [PMID:18075950] http://dx.doi.org/10.1682/JRRD.2007.01.0003

14. Green W, Ciuffreda KJ, Thiagarajan P, Szymanowicz D, Ludlam DP, Kapoor N. Accommodation in mild traumatic brain injury. J Rehabil Res Dev. 2010;47(3):183-99. [PMID:20665345] http://dx.doi.org/10.1682/JRRD.2009.04.0041

15. Halbauer JD, Ashford JW, Zeitzer JM, Adamson MM, Lew HL, Yesavage JA. Neuropsychiatric diagnosis and management of chronic sequelae of war-related mild to moderate traumatic brain injury. J Rehabil Res Dev. 2009;46(6):757-96. [PMID:20104402] http://dx.doi.org/10.1682/JRRD.2008.08.0119

16. Reger MA, Gahm GA, Swanson RD, Duma SJ. Association between number of deployments to Iraq and mental health screening outcomes in US Army soldiers. J Clin Psychiatry. 2009;70(9):1266-72. [PMID:19689917] http://dx.doi.org/10.4088/JCP.08m04361

17. Rona RJ, Fear NT, Hull L, Greenberg N, Earnshaw M, Hotopf M, Wessely S. Mental health consequences of overstretch in the UK armed forces: first phase of a cohort study. BMJ. 2007;335(7620):603. [PMID:17664192] http://dx.doi.org/10.1136/bmj.39274.585752.BE
18. Fear NT, Jones M, Murphy D, Hull L, Iversen AC, Coker B, Machell L, Sundin J, Woodhead C, Jones N, Greenberg N, Landau S, Dandeker C, Rona RJ, Hotopf M, Wessely S. What are the consequences of deployment to Iraq and Afghanistan on the mental health of the UK armed forces? A cohort study. Lancet. 2010;375(9728):1783-97. [PMID:20471076] http://dx.doi.org/10.1016/S0140-6736(10)60672-1

19. Hoffman SW, Harrison C. The interaction between psychological health and traumatic brain injury: a neuroscience perspective. Clin Neuropsychol. 2009;23(8):1400-1415. [PMID:19882478] http://dx.doi.org/10.1080/13854040903369433

20. Konrad C, Geburek AJ, Rist F, Blumenroth H, Fischer B, Husstedt I, Arolt V, Schiffbauer H, Lohmann H. Long-term cognitive and emotional consequences of mild traumatic brain injury. Psychol Med. 2010;22:1-15. [PMID:20860865]

21. Stulemeijer M, Vos PE, van der Werf S, van Dijk G, Rijpkema M, Fernández G. How mild traumatic brain injury may affect declarative memory performance in the postacute stage. J Neurotrauma. 2010;27(9):1585-95. [PMID:20629484] http://dx.doi.org/10.1089/neu.2010.1298

Submitted for publication February 9, 2011. Accepted in revised form June 17, 2011.

This article and any supplementary material should be cited as follows:

Bulson R, Jun W, Hayes J. Visual symptomatology and referral patterns for Operation Iraqi Freedom and Operation Enduring Freedom veterans with traumatic brain injury. J Rehabil Res Dev. 2012;49(7):1075-82. http://dx.doi.org/10.1682/JRRD.2011.02.0017

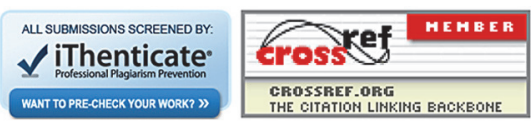

